\title{
Hipoglucemia por trimetoprim-sulfametoxazol
}

Trimethoprim-sulfamethoxazole hypoglycemia

Hipoglicemia de trimetoprim-sulfametoxazol

\author{
doi) http://dx.doi.org/10.35954/SM2020.39.2.9 \\ Víctor Edelstein Zarucki a \\ María Florencia Segovia Cervetti ${ }^{b}$ \\ https://orcid.org/0000-0001-8573-9936 \\ https://orcid.org/0000-0003-0989-0452 \\ (a) Departamento de Medicina, Servicio de Endocrinología, Hospital Central de las Fuerzas Armadas. \\ (b) Departamento de Medicina, Servicio de Medicina Interna, Hospital Central de las Fuerzas Armadas.
}

\section{RESUMEN}

La hipoglucemia es una complicación frecuente en pacientes diabéticos. En la mayoría de los casos ocurre en relación al tratamiento con antidiabéticos o el uso de insulina. Es importante su prevención y su tratamiento inmediato, dado las complicaciones graves que pueden ocurrir como consecuencias de las mismas, sobre todo la elevada mortalidad y secuelas neurológicas y cardíacas. La hipoglucemia también ocurre por fármacos diferentes a los involucrados en el tratamiento antidiabético. Se analizó el caso clínico de una paciente portadora de Diabetes Mellitus tipo 2 y Mieloma Múltiple, que presentó una complicación infecciosa y respiratoria de la poliquimioterapia. Requirió tratamiento con TrimetropimSulfametoxazol, el cual produjo como efecto adverso hipoglucemias, en una paciente que tenía factores predisponentes para su desarrollo y además en el contexto de interacciones medicamentosas. Luego de descender la dosis del antibiótico, no reiteró hipoglucemias, con buena evolución posterior. La hipoglicemia por Trimetoprim-Sulfametoxazol es un efecto adverso poco frecuente. Se ve favorecida en pacientes que presentan factores predisponentes para su desarrollo; en quienes reciben múltiples fármacos, se debe tener en cuenta las interacciones farmacológicas y posibles efectos adversos, al agregar un nuevo medicamento como se describe en el presente caso.

PALAVRAS CLAVE: Combinacion Trimetoprim y Sulfametoxazol; Diabetes Mellitus Tipo 2; Hipoglucemia; Infecciones por Pneumocystis; Insuficiencia Renal; Mieloma Múltiple; Neumonia por Pneumocystis; Pneumocystis carinii; Pneumocystis jirovecii.

\section{ABSTRACT}

Hypoglycemia is a frequent complication in diabetic patients. In most cases it occurs in relation to treatment with antidiabetic drugs or the use of insulin. Its prevention and immediate treatment is important, given the serious complications that can occur as a consequence, especially high mortality and neurological and cardiac sequelae. Hypoglycemia also occurs due to drugs other than those involved in antidiabetic treatment. We analyzed the clinical case of a patient with type 2 Diabetes Mellitus and Multiple Myeloma, who presented an infectious and respiratory complication of polychemotherapy. She required 
treatment with Trimetropim-Sulfamethoxazole, which produced hypoglycemia as an adverse effect, in a patient who had predisposing factors for its development and also in the context of drug-drug interactions. After lowering the antibiotic dose, she did not repeat hypoglycemias, with good subsequent evolution. Hypoglycemia due to Trimethoprim-Sulfamethoxazole is an infrequent adverse effect. It is favored in patients who present predisposing factors for its development; in those who receive multiple drugs, pharmacological interactions and possible adverse effects should be taken into account when adding a new drug as described in the present clinical case.

KEY WORDS: Trimethoprim, Sulfamethoxazole Drug Combination; Diabetes Mellitus Type 2; Hypoglycemia; Pneumocystis Infections; Renal Insufficiency; Multiple Myeloma; Pneumonia, Pneumocystis;

Pneumocystis carinii; Pneumocystis jirovecii.

\section{RESUMO}

A hipoglicemia é uma complicação freqüente em pacientes diabéticos. Na maioria dos casos ocorre em relação ao tratamento com medicamentos antidiabéticos ou ao uso de insulina. Sua prevenção e tratamento imediato é importante, dadas as sérias complicações que podem ocorrer como conseqüência, especialmente a alta mortalidade e as seqüelas neurológicas e cardíacas. A hipoglicemia também ocorre devido a outros medicamentos que não aqueles envolvidos no tratamento antidiabético. Analisamos o caso clínico de um paciente com Diabetes Mellitus tipo 2 e Mieloma Múltiplo, que apresentava uma complicação infecciosa e respiratória da poli-quimioterapia. Ela requereu tratamento com Trimetropim-Sulfamethoxazole, que produziu hipoglicemia como efeito adverso, em um paciente que tinha fatores predisponentes para seu desenvolvimento e também no contexto das interações medicamentosas. Após baixar a dose de antibióticos, ela não repetiu as hipoglicemias, com boa evolução posterior. A hipoglicemia devida ao Trimethoprim-Sulfamethoxazol é um efeito adverso pouco freqüente. Ela é favorecida nos pacientes que apresentam fatores predisponentes para seu desenvolvimento; naqueles que recebem múltiplos medicamentos, as interações farmacológicas e possíveis efeitos adversos devem ser levadas em consideração ao adicionar um novo medicamento, conforme descrito no presente caso clínico.

PALAVRAS ChaVE: Combinação Trimetoprima e Sulfametoxazol; Diabetes Mellitus Tipo 2; Hipoglicemia; Infecções por Pneumocystis; Insuficiência Renal; Mieloma Multiplo; Pneumonia por Pneumocystis; Pneumocystis carinii; Pneumocystis jirovecii.

\section{INTRODUCCIÓN}

La diabetes mellitus tipo 2 es una enfermedad de alta prevalencia en el mundo. Frecuentemente asistimos a la aparición de hipoglucemias, complicación que puede llegar a ser fatal. La misma puede ser consecuencia del tratamiento antidiabético, ya sea con antidiabéticos orales o con insulina, o ambos tratamientos combinados. Esto es debido a la tendencia a lograr un mejor y estricto control metabólico con el fin de evitar o retrasar las complicaciones propias de la diabetes mellitus. El he- cho que los pacientes con diabetes mellitus sean más propensos a desarrollar cuadros infecciosos que requieren diferentes tratamientos antimicrobianos determina que la frecuencia de hipoglucemia se vea acrecentada como reacción adversa a los mismos (1).

Uno de estos fármacos es el Trimetoprim-Sulfametoxazol (TMP-SMX), un antibiótico ampliamente usado en la práctica clínica habitual, si bien al inicio fue diseñado para su uso en infecciones del tracto urinario, actualmente se emplea para prevención y tratamiento de múltiples infecciones entre ellas las 
infecciones causadas por pneumocysitis jiroveci (2). Si se trata de un paciente hospitalizado el riesgo de hipoglucemia es mayor debido a la variabilidad en la sensibilidad a la insulina relacionada con la enfermedad subyacente.

\section{CASO CLÍNICO}

Paciente de 64 años, que ingresa desde policlínica dado que comienza el día previo con fiebre, tos irritativa y aumento de su disnea habitual. Sin síntomas cardiovasculares, sin hemoptisis. Como antecedentes personales a destacar presenta: hipertensión arterial, hipotiroidismo, diabetes mellitus tipo 2, obesidad, hipercolesterolemia, síndrome de apnea obstructiva del sueño, trombosis venosa profunda en miembro inferior izquierdo y trombosis venosa profunda de miembro inferior derecho. Anticoagulada con warfarina por fibrilación auricular y tromboembolismo pulmonar. Mieloma múltiple. Recibe tratamiento con: $\mathrm{T} 4$, digoxina, metformina, diltiazem, allopurinol, warfarina, b2 inhalatorios, y combinación de ciclofosfamida, vincristina, adriamicina y dexametasona (CVAD).

Al examen físico se encontraba: lúcida, frecuencia respiratoria 20 rpm, saturación $\mathrm{O} 2$ ventilando espontáneamente al aire $94 \%$.

Piel y mucosas: normocoloreadas

Cardiovascular: ritmo regular 88 ciclos por minuto, ruidos bien golpeados, sin soplos.

Pleuropulmonar: murmullo alvéolo vesicular presente bilateralmente, estertores bilaterales tipo velcro y estertores secos.

Miembros inferiores: sin edemas.

Se diagnosticó en primera instancia neumonia aguda, la cual puede ser causada por gérmenes inespecíficos, o en el caso de la paciente, por haber recibido quimioterapia previamente, podría tratarse de una neumopatía específica, como por ejemplo tuberculosis o aspergilosis. Como segundo planteo cabe la posibilidad de que se tratase de un nuevo tromboembolismo pulmonar. En tercer lugar, pero más alejado habría que pensar en una intersticiopatia de base con sobreinfección. Realizados los exámenes clínicos pertinentes, advierten que presenta hemoglobina: $10 \mathrm{~g} / \mathrm{dl}$, plaquetas 355000/mm3, glóbulos blancos 6800/mm3, neutrófilos 4200/mm3.

Azoemia $63 \mathrm{mg} / \mathrm{dl}$, creatininemia 1,5 mg/dl. lonograma: Na $130 \mathrm{mEq} / \mathrm{l}$ y K 4,8 mEq/l.

Se realizó una tomografía axial computada, que evidenció una intersticiopatía tipo neumonia organizativa criptogenética o a neumonia intersticial aguda. Posteriormente mediante una fibrobroncoscopía con lavado bronquioalveloar surge el hallazgo de pneumocystis jirovecii positivo. Resultando negativa para tuberculosis.

$\mathrm{Al}$ inicio recibió tratamiento antibiótico en base a ceftriaxona. Posteriormente valorada por infectólogo, se ajusta el mismo a dosis de 3 ampollas cada 8 horas (cada ampolla contiene 160 mg de trimetropim y $800 \mathrm{mg}$ de sulfametoxazol) cambiando su administración de vía oral a vía intravenosa. Al ser internada en sala permanece en tratamiento con dieta de diabético más ajustes con insulina cristalina subcutánea para tratar excursiones de hiperglucemias. Previamente a su internación, la paciente estaba bajo tratamiento con metformina a dosis de 1700 mg día, ésta fue suspendida ante la presencia de insuficiencia renal, presentando un clearence de creatinina disminuído.

Dada la persistencia del descontrol metabólico hiperglicémico, se realizó consulta con diabetólogo, quien agregó glimepirida a bajas dosis de $2 \mathrm{mg} /$ día vía oral. Inmediatamente presentó un episodio de hipoglucemia sintomática. Si bien existían otros factores que contribuían a la hipoglicemia, tales como la insuficiencia renal y el estado nauseoso que determinaba la baja ingesta calórica, se planteó la posibilidad que la glimepirida a bajas dosis pudiera ser la causa de las hipoglucemias. Es así que inmediatamente se suspende la glimepirida, manteniendo tratamiento antidiabético, únicamente con dieta más ajustes con insulina cristalina por vía subcutánea. Aun así, cuatro días después, las hipoglucemias persistían oscilando 
en valores de entre 45 a $63 \mathrm{mg} / \mathrm{dl}$.

Las mismas requirieron tratamiento con suero glucosado intravenoso en varias oportunidades. Algo llamativo fue que no requirió muchos ajustes con insulina cristalina, lo cual, de haber ocurrido podría explicar los episodios de hipoglicemia por la mayor vida media de la misma en pacientes con insuficiencia renal. Estos hechos alertaron al equipo médico sobre otras posibles causas que la explicaran, realizándose un relevo de todos los medicamentos con los que estaba siendo tratada. Uno de ellos era el antibiótico TMP-SMX. Este fármaco tiene dentro de sus efectos adversos la ocurrencia de hipoglucemia, aunque se describe como poco frecuente. Se disminuyó la dosis, con lo cual no reiteró hipoglucemias. Por lo tanto se planteó una relación causa-efecto y temporal asociado al uso de este antibiótico y la presencia de hipoglucemias.

\section{DISCUSIÓN}

La hipoglucemia es una complicación que puede ser fatal en todo paciente diabético. En presencia de diagnóstico confirmado de diabetes, la hipoglicemia se define como todo episodio con una concentración de glucosa plasmática anormalmente baja que exponga al individuo a un daño potencial y se establece un valor de glicemia menor de $70 \mathrm{mg} / \mathrm{dl}$ (1). En este caso debemos analizar dos elementos diferentes: el mecanismo mediante el cual el TMPSMX puede producir hipoglucemias y los factores predisponentes hallados en esta paciente. TMP-SMX es una combinación de dos principios activos, el sulfametoxazol (una sulfonamida o sulfamida) y el trimetoprim. Su administración en conjunto produce una sinergia atribuida a la inhibición de la producción de tetrahidrofolato, lo cual promueve el desarrollo más lento de resistencia bacteriana (3).

El mecanismo de acción propuesto como causa de hipoglucemia por este antibiótico, está basado en que pertenece al grupo de las sulfonamidas, con semejanza bioquímica con las sulfonilureas (uno de los grupos de antidiabéticos orales). La unión del sulfametoxazol al receptor de la célula beta de los islotes pancreáticos, aumenta la secreción de insulina (4).

Prueba de ello es el hallazgo de dosificaciones más altas de insulina plasmática, al igual que del péptico $C$ en quienes usaban este antibiótico y presentaron hipoglucemia. Por lo tanto se trataban de hipoglucemias hiperinsulinémicas $(5,6)$. Cabe destacar que no fue necesario dosificar la insulinemia y hubiese sido erróneo hacerlo dado que la paciente recibía insulina exógena.

A esta similitud del mecanismo de acción del TMP-SMX, a nivel de la célula beta de los islotes pancreáticos se le denomina "efecto sulfonilurea-like" $(7,8)$.

EI TMP-SMX se elimina sobre todo por vía renal, después de metabolizarse a nivel hepático. La vida media de eliminación del sulfametoxazol varía entre 6 y 12 horas si la función renal está conservada, y entre las 20 y 50 horas cuando existe insuficiencia renal, como ocurrió en esta paciente. En caso de presentar insuficiencia renal, se debe reducir la dosis, ajustándola al clearence de creatinina. Por lo tanto el deterioro de la función renal es el factor desencadenante más importante, ya que aumenta la vida media de los fármacos que se eliminan por esta vía (5).

Debido a la infección respiratoria que portaba la paciente, se usaron dosis altas y por vía intravenosa. Ello constituye un factor predisponente muy importante ante la existencia de insuficiencia renal. Al mecanismo de hipoglucemia producido por el TMP-SMX, en este caso clínico se suman varios factores predisponentes.

Al presentar insuficiencia renal, la vida media de muchos medicamentos se prolonga, como en el caso de la glimepirida, que además por sí misma, es otro factor predisponente para producir hipoglucemias, dado que es un antidiabético oral del grupo de las sulfonilureas, que tiene como mecanismo de acción aumentar la secreción de insulina. La vida media de la insulina cristalina, utilizada 
para la corrección de las hiperglicemias, también se ve incrementada en la insuficiencia renal, Ilevando a la misma consecuencia. A estos hechos se suma la escasa ingesta calórica debido al estado nauseoso que presentaba.

Se reportan casos de hipoglucemias favorecidas por TMP-SMX en pacientes desnutridos o con baja ingesta calórica y sobre todo con infecciones severas (9).

En otras sulfonilureas como la glibenclamida y la glipizida (esta última no presente en Uruguay), se plantea otro tipo de interacción con el TMP-SMX. Estas sulfonilureas se metabolizan por una enzima hepática dentro del gran grupo citocromo p450 (CYP 450).

EI TMP-SMX inhibe una de estas enzimas, la CYP 2C9, por lo tanto disminuiría el metabolismo hepático de estos antidiabéticos orales. Si bien en la literatura no encontramos que esta enzima esté relacionada con el metabolismo de la glimepirida, podrían existir analogías entre el metabolismo hepático y todas las sulfonilureas, lo cual podría ser una hipótesis a investigar (4,10-12).

DECLARACIÓN DE CONFLICTOS DE INTERESES: Los autores no reportan ningún conflicto de interés. El estudio se realizó con recursos propios de los autores y/o la institución a la que representan.

\section{REFERENCIAS}

(1) García de los Ríos M, Durruty P. Diabetes Mellitus. 3a.ed.

Santiago de Chile : Mediterráneo, 2014. p. 151-157.

(2) Agencia española de medicamentos y productos sanitarios.

Ficha técnica SOLTRIM 160mg/ 800mg POLVO Y SOLUCION PARA SOLUCION INYECTABLE. Disponible en: https://cima.aemps.es/cima/pdfs/es/ ft/54920/FT_54920.pdf [Consulta 19/07/2020].

(3) Petri WA Jr. Sulfonamidas, trimetoprim-sulfametoxazol, quinolonas y fármacos contra infecciones de las vías urinarias. En: Brunton L, Lazo J, Parker K, Eds. Goodman y Gilman. Las bases farmacológicas de la terapéutica. Sección VIII. Cap.43. 11a.ed. México : McGraw Hill, 2006. p.1111-1125.

(4)EshraghianA, Omrani G. Cotrimoxazole-induced hypoglycemia in outpatient setting.

Nutrition 2014; 30(7-8):959.

doi:10.1016/j.nut.2014.01.002

(5) Caro J, Navarro-Hidalgo I, Civera M, Real J, Ascaso J. Hipoglucemia grave y prolongada secundaria a cotrimoxazol en sujeto con factores predisponentes.

Endocrinol Nutr 2012; 59(2):146-8.

doi: 10.1016/j.endonu.2011.07.012

(6) Chan J, Cockram C, Critchley J. Drug-induced disorders of glucose metabolism. Mechanisms and management. Drug Saf 1996; 15(2):135-157. doi: 10.2165/00002018-199615020-00005

(7) Okazaki M, Okazaki M, Nakamura M, Asagiri T, TakeuchiS. Consecutive hypoglycemiaattacks induced by co-trimoxazole followed by pentamidine in a patient with acquired immunodeficiency syndrome. Int J STD AIDS 2019; 30(1):86-89. doi: $10.1177 / 0956462418795580$

(8) Nunnari G, Celesia BM, Bellissimo F, Tosto S, La Rocca M, Giarratana F, et al. Trimethopim-Sulfamethoxazole-associated severe hypoglycemia: a Sulfonylurea-like effect.

Eur Rev Med Pharmacol Sci 2010; 14(12):1015-8. 
(9) Hekimsoy Z, Biberoglu S, Comlecki A, Tarhan O, Mermut C, Biberoglu K.

Trimethoprim/sulfamethoxazole-induced hypoglycemia in a malnourished patient with severe infection. Eur J Endocrinol 1997; 136(3):304-6.

doi: 10.1530/eje.0.1360304

(10) Tan A, Holmes HM, Kuo Y, Raji M, Goodwin J. Coadministration of co-trimoxazole with sulfonylureas: hypoglycemia events and pattern of use. J Gerontol Series A 2015; 70(2):247-254.

https://doi.org/10.1093/gerona/glu072

(11) Parekh T, Raji M, Lin Y, Tan A, Kuo Y, Goodwin J. Hypoglycemia after antimicrobial drug prescription for older patients using sulfonylureas. JAMA Intern Med 2014; 174(10):1605-1612.

doi:10.1001/jamainternmed.2014.3293

(12) Schelleman H, Bilker W, Brensinger C, Wan F, Hennessy S. Anti-infectives and risk of severe hypoglycemia in glipizide and glyburide users. Clin Pharmacol Ther 2010; 88(2):214-222.

doi:10.1038/clpt.2010.74 